\title{
Protective population behavior change in outbreaks of emerging infectious disease
}

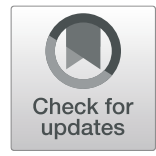

Evans K. Lodge $\mathrm{e}^{1,2^{*}} \mathbb{D}$, Annakate M. Schatz ${ }^{3}$ and John M. Drake ${ }^{3}$

\begin{abstract}
Background: During outbreaks of emerging and re-emerging infections, the lack of effective drugs and vaccines increases reliance on non-pharmacologic public health interventions and behavior change to limit human-tohuman transmission. Interventions that increase the speed with which infected individuals remove themselves from the susceptible population are paramount, particularly isolation and hospitalization. Ebola virus disease (EVD), Severe Acute Respiratory Syndrome (SARS), and Middle East Respiratory Syndrome (MERS) are zoonotic viruses that have caused significant recent outbreaks with sustained human-to-human transmission.

Methods: This investigation quantified changing mean removal rates (MRR) and days from symptom onset to hospitalization (DSOH) of infected individuals from the population in seven different outbreaks of EVD, SARS, and MERS, to test for statistically significant differences in these metrics between outbreaks.

Results: We found that epidemic week and viral serial interval were correlated with the speed with which populations developed and maintained health behaviors in each outbreak.

Conclusions: These findings highlight intrinsic population-level changes in isolation rates in multiple epidemics of three zoonotic infections with established human-to-human transmission and significant morbidity and mortality. These data are particularly useful for disease modelers seeking to forecast the spread of emerging pathogens.
\end{abstract}

Keywords: Infectious disease, Outbreak, Public health, Ebola, SARS, MERS

\section{Background}

One of the most important factors in assessing the health risks posed by an epidemic of infectious disease is pathogen transmissibility. Widespread public anxiety during the 2013-2016 West African Ebola epidemic, while driven by an extremely high fatality rate during the early stages, was fueled in part by the speed with which Ebola Virus Disease (EVD) spread throughout the populations of Liberia, Sierra Leone, and Guinea [1-3]. Similarly, concerns over annual influenza epidemics in

\footnotetext{
* Correspondence: evans_lodge@med.unc.edu

'Department of Epidemiology, Gillings School of Global Public Health, University of North Carolina at Chapel Hill, 135 Dauer Drive, Chapel Hill, NC 27599, USA

${ }^{2}$ School of Medicine, University of North Carolina at Chapel Hill, Chapel Hill, North Carolina, USA

Full list of author information is available at the end of the article
}

the United States center on densely inhabited areas with multiple opportunities for viral transmission due to physical proximity between susceptible individuals [4]. Regardless of geographic setting, understanding how to slow and control pathogen dissemination is a high priority in forecasting and preventing epidemics of infectious disease.

Epidemic modelers frequently employ compartmental models of disease outbreaks, such as SusceptibleInfected-Recovered (SIR) models as in Keeling and Rohani [5], Susceptible-Infected-Susceptible (SIS) models as in Gray et al [6], and Susceptible-ExposedInfected-Recovered (SEIR) models as in LeGrand et al [7]. Accurately estimating and modeling the number of infected and susceptible individuals in at-risk populations is of crucial importance in these models. Such estimation is complicated, however, by efforts to isolate

(c) The Author(s). 2021 Open Access This article is licensed under a Creative Commons Attribution 4.0 International License, which permits use, sharing, adaptation, distribution and reproduction in any medium or format, as long as you give appropriate credit to the original author(s) and the source, provide a link to the Creative Commons licence, and indicate if changes were made. The images or other third party material in this article are included in the article's Creative Commons licence, unless indicated otherwise in a credit line to the material. If material is not included in the article's Creative Commons licence and your intended use is not permitted by statutory regulation or exceeds the permitted use, you will need to obtain permission directly from the copyright holder. To view a copy of this licence, visit http://creativecommons.org/licenses/by/4.0/ The Creative Commons Public Domain Dedication waiver (http://creativecommons.org/publicdomain/zero/1.0/) applies to the data made available in this article, unless otherwise stated in a credit line to the data. 
infected individuals in healthcare facilities or other community settings to decrease contact with the susceptible population. While the isolation of infected individuals is beneficial and should be encouraged, it challenges data analysts because it is time-varying and reflects dynamic and often unpredictable human behavior. Moreover, the rate at which infected individuals are removed from the population typically accelerates throughout an epidemic as awareness of the infectious threat increases [8], a process Drake et al referred to as "societal learning" [9]. Obtaining accurate estimates of this time-varying removal of infected persons, while difficult, improves the quality of compartmental models for epidemics of infectious disease $[9,10]$. To our knowledge, however, no work has directly compared the rate of behavioral adaption across multiple epidemics, societies, and geographic settings.

Many factors can affect how quickly effective isolation practices are implemented, such as access to health care, local public health funding, international aid, and the efficacy of information campaigns [11]. Local health care practices and non-formal healthcare systems also provide care to patients during epidemics and can play a part in quarantining infected individuals [12]. Previous work in Liberia has shown that a combination of these approaches through simultaneous community engagement and clinical intervention is more effective than any single intervention, with both health care access and utilization increasing hand-in-hand to decrease EVD transmission during the 2013-2016 Ebola epidemic [13]. While infection prevention and control practices often include vaccination, progress to develop effective vaccines for emerging infections is slow and not necessarily more effective than isolation of infected individuals [14]. Ring vaccination with the rVSV-ZEBOV-GP Ebola vaccine [15] in the Democratic Republic of the Congo is promising [16], but previous work has suggested that ring vaccination may only provide a marginal benefit to rigorous contact tracing and patient isolation [17].

This paper aims to identify key similarities and differences in the behavioral response to outbreaks of three emerging zoonotic infections, focusing particularly on the speed with which infected individuals are removed from the susceptible population. We sought to determine how the mean removal rate (the inverse of the number of days from symptom onset to isolation of infected individuals) changed over the course of each outbreak as measured by epidemic week and viral serial interval. Individuals often experience zoonotic and emerging infections as innately more frightening than "familiar" diseases, leading to rapid behavioral adaptations due to high perceived risk of severe disease and death [18]. Behavior modification, while crucial for epidemic containment [19-21], is context dependent and difficult to predict due to social network, socioeconomic, and behavioral differences between populations [22]. Thus, we chose seven different outbreaks of disease that stoked significant local and international fear due to the risk of global pandemic: the 2013-2016 Liberian Ebola epidemic, subsets of the 2013-2016 Liberian outbreak from Lofa and Montserrado Counties [23], the 2003 Hong Kong SARS epidemic [24-26], the 2014 Saudi Arabia MERS outbreaks in Riyadh and Jeddah [27], and the 2015 South Korea MERS outbreak [28]. We examined whether epidemic week and serial interval successfully predicted days from disease onset to hospitalization (DSOH) and mean removal rate (MRR) throughout each epidemic.

\section{Methods \\ Data}

We obtained anonymous patient-level data for Ebola and MERS, and daily aggregated data for SARS. Requirements for participant consent were waived by the Institutional Review Board (IRB) of the University of Georgia (Protocol ID\#STUDY00002338). We added new columns to each epidemic dataset to track the number of days from symptom onset to hospitalization (calculated as hospitalization date - date of symptom onset; abbreviated DSOH) and the mean removal rate (calculated as 1 / DSOH; abbreviated MRR). In calculating MRR, we considered only positive DSOH values in order to focus on community transmission rather than nosocomial transmission. Additionally, we converted symptom onset dates to weekly onset dates by replacing each date with that of the closest previous Sunday. We used weekly onset dates to aggregate data by epidemic week as described below.

\section{Binned data}

We compiled data for each outbreak location binned by epidemic week, to produce comparable data for regression analysis. Epidemic weeks came from weekly onset dates described above. We also binned the same data by serial interval, using 12 days as the estimated serial interval for Ebola [23], 8 days for SARS [24], and 7 days for MERS [27]; this was calculated as epidemic week/(serial interval/7). Each dataset included, per week, the number of new cases, the cumulative number of cases, mean DSOH and associated standard deviation, and MRR and associated standard deviation. We removed epidemic weeks from the beginning of each outbreak so that the first three epidemic weeks had greater than 0 cases of disease each in order to focus on population-level behavioral adaptation to large-scale disease outbreaks instead of adaptations to individual disease events early in an epidemic. We performed all regression analyses using 
this binned data, weighted by the number of cases per epidemic week.

\section{Regression analyses}

Initial regression analyses fit linear models to predict DSOH and MRR (Table 1, Eqs. 1-2). As before, data for $\mathrm{DSOH}$ excluded negative values (individuals who become symptomatic after being hospitalized for other reasons) to focus on community disease transmission and behavior change instead of nosocomial infection.

Outlying points in the Liberian Ebola epidemic skewed our initial linear regression models. We compared manual removal of outliers, quantile regression, and robust linear regression to find the most appropriate method for handling such points. The three methods produced almost identical results. We used robust regression to re-fit all initial linear regression models to avoid the influence of outliers (Table 1, Eqs. 3-4). In addition, we performed robust linear regressions of MRR with an interaction term accounting for outbreak location (Table 1, Eq. 5-6) to examine predicted mean change in the MRR in each epidemic. We used the Bonferroni correction [29] for multiple comparisons to compute confidence intervals, utilizing a $99 \%$ confidence interval in our model comparisons. The size of the smaller epidemics (MERS and SARS) played a large part in determining confidence interval size and significance. All data management, modeling, and visualization was performed in $\mathrm{R}[30]$.

\section{Results}

\section{Days to hospitalization (DSOH) and mean removal rate (MRR)}

DSOH consistently declined over time in each epidemic. Robust regressions for DSOH and MRR (Table 1, Eqs. 3 and 4) showed negative and positive slopes, respectively, which corroborated the observations made on nonbinned data (Fig. 1). Complete regression tables are provided in online supplementary materials.

Table 1 Regression equations predicting DSOH and MRR

\begin{tabular}{lllll}
\hline Eq. & Regression Type & Response & Predictor & Interaction Term \\
\hline $\mathbf{1}$ & linear & DSOH & epidemic week & none \\
$\mathbf{2}$ & linear & MRR & epidemic week & none \\
$\mathbf{3}$ & robust linear & DSOH & epidemic week none \\
$\mathbf{4}$ & robust linear & MRR & epidemic week none \\
$\mathbf{5}$ & robust linear & MRR & epidemic week & outbreak location \\
$\mathbf{6}$ & robust linear & MRR & serial interval & outbreak location
\end{tabular}

$\mathrm{DSOH}$ stands for days from symptom onset to hospitalization, MRR stands for mean removal rate, calculated as (1 / DSOH). Epidemic weeks were weighted by cases per week. Outbreak location for DSOH and MRR included seven levels (Liberia, Lofa County, Montserrado County, South Korea, Riyadh, Jeddah, and Hong Kong)
From regression analyses accounting for outbreak location (Table 1, Eqs. 5 and 6), we calculated the mean change in the MRR for each outbreak location using the interactionMeans function from the $\mathrm{R}$ package phia for post-hoc interaction analysis. This analysis showed that the mean change in the MRR of the Hong Kong SARS epidemic was approximately five times (per serial interval) to seven times (per epidemic week) more than the mean change in the MRR of the Liberian Ebola epidemic (Fig. 2). The mean change of the MRR in the Ebola epidemic in Lofa County, Liberia, was significantly higher than the mean change of the MRR for the overall Liberian epidemic and the outbreak in Montserrado County, Liberia, regardless of predictor (epidemic week or serial interval) (Fig. 2). The three MERS outbreaks (Riyadh, Jeddah, and South Korea) did not differ significantly from one another and had limited precision (Fig. 2).

We found that predicting mean change of the MRR by epidemic week (Table 1, Eq. 5) led to higher mean estimates and wider confidence intervals in the MERS and SARS outbreaks; conversely, predicting with serial interval (Table 1, Eq. 6) lowered mean estimates and narrowed the associated confidence intervals (Fig. 2). We identified little difference in the mean change of the MRR for the Ebola outbreak depending on predictor (Fig. 2). This indicates that, at least in the case of MERS and SARS, both the passage of time and the serial interval of each virus may affect the speed with which populations develop and maintain health behaviors.

\section{Discussion}

The models presented in this study show that the removal of infected individuals from the susceptible population increases over time and varies significantly based on outbreak duration and location. Our findings suggest that populations perceive the health risks posed by emerging and re-emerging pathogens and respond rapidly to limit community transmission. Such populationlevel quarantine behaviors develop quickly in extremely disparate settings (Liberia, Hong Kong, Saudi Arabia, and South Korea) and distinct viral outbreaks (SARS, MERS, and Ebola). While DSOH improved (decreased) in every epidemic over time, extreme disparities in starting values (approximately 13 days from symptom onset to hospitalization at the beginning of the 2013-2016 Ebola outbreak in Liberia, versus approximately 5 days in the 2015 MERS outbreak in South Korea) highlight the intrinsic disadvantage that low-income countries may experience due to the interrelated concerns of poverty, limited access to health care, and low investment in public health. DSOH and MRR regressed against epidemic week differed across all observed outbreaks, and MRR likewise differed markedly based on the virus in question (Ebola, MERS, or SARS), the location, and at 

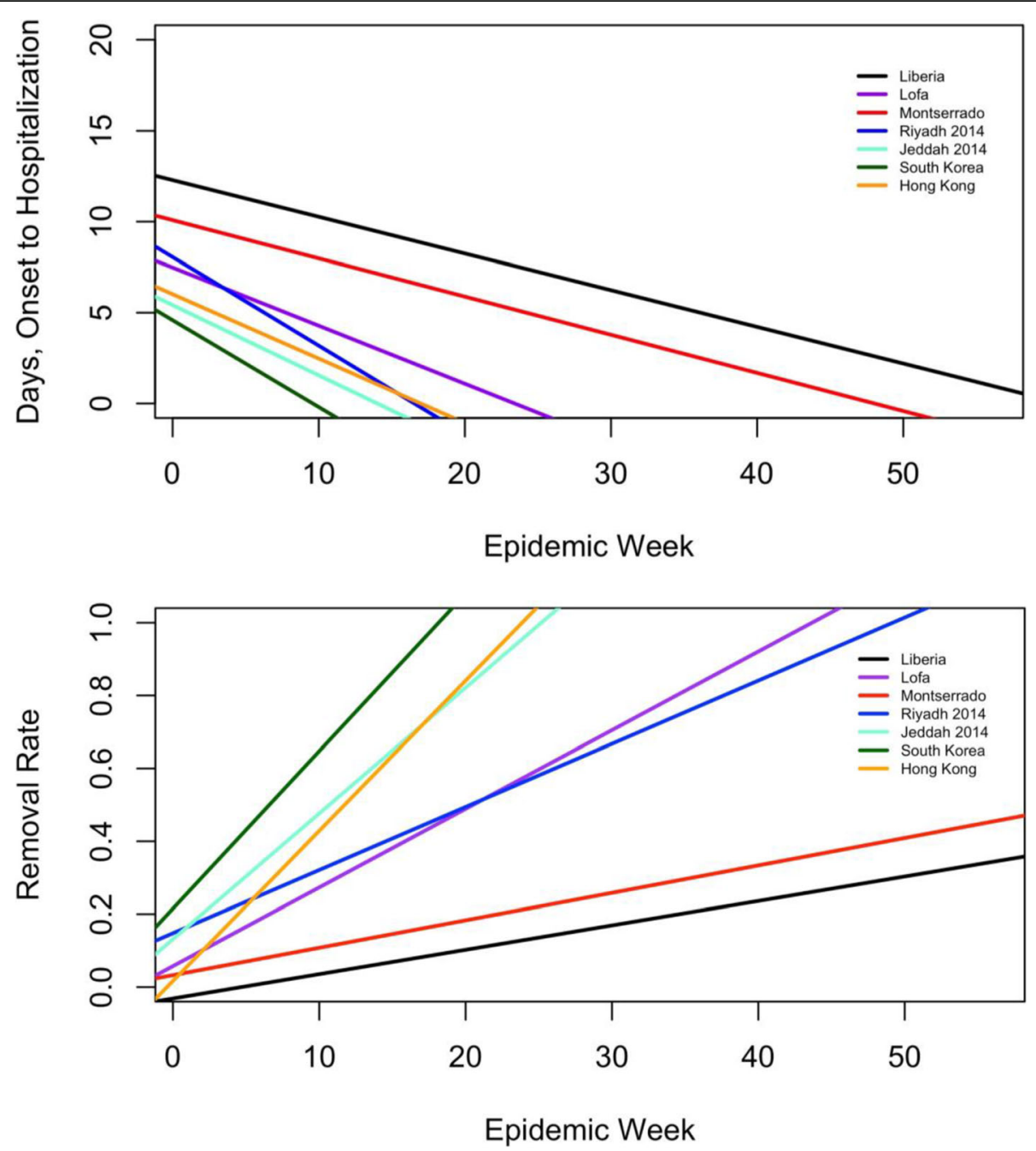

Fig. 1 Regression results predicting DSOH and MRR by epidemic week. The public health response to epidemic infection varied widely between the outbreaks studied. These graphs depict model lines from regressions of each of the 2 response variables (DSOH (Table 1, Eq. 4) and MRR (Table 1, Eq. 5)) on epidemic week for the 7 outbreaks indicated in the legend. South Korea and Liberia exhibited the most extreme slopes in both analyses. As an illustration of the observed difference between outbreaks, the graphs show South Korea achieving an almost complete removal of infected individuals from the population and a sharp decline in days till hospitalization within 20 weeks, while Liberia only achieved a roughly $20 \%$ removal rate by 50 weeks

times both. Both DSOH and MRR are useful measurements of public health behavior during outbreaks, and are useful tools to compare outbreak response effectiveness in distinct geographic, economic, and social settings. Of course, DSOH and MRR are intrinsically and simply related since one is simply the reciprocal of the other. The main advantage of $\mathrm{DSOH}$ is that it is expressed in intuitive units (days elapsed), whereas MRR reflects the theoretical "removal rate" of standard compartmental models [5].

Figure 2 highlights differences in mean change of the MRR due to outbreak type (Ebola, MERS, or SARS) and location. Mean change of the MRR was similar when calculated using epidemic week versus serial interval for Ebola, but demonstrated a lower estimate and lower standard error when calculated using serial interval in all three outbreaks of MERS and the outbreak of SARS in Hong Kong. This suggests that the relevance of various predictors (epidemic week versus serial interval) may vary based upon the type and location of an outbreak, although the comparative relevance of epidemic type versus location cannot be disentangled with the data available in this study. We recommend similar analyses of MRR be conducted across a wide range of geographies as outbreaks of emerging pathogens arise, providing important data on the range of MRR, and its expected rate of change, in different settings. This approach would be further strengthened by incorporating data regarding other context- and culture-specific social conditions, such as the presence of domestic and international aid 


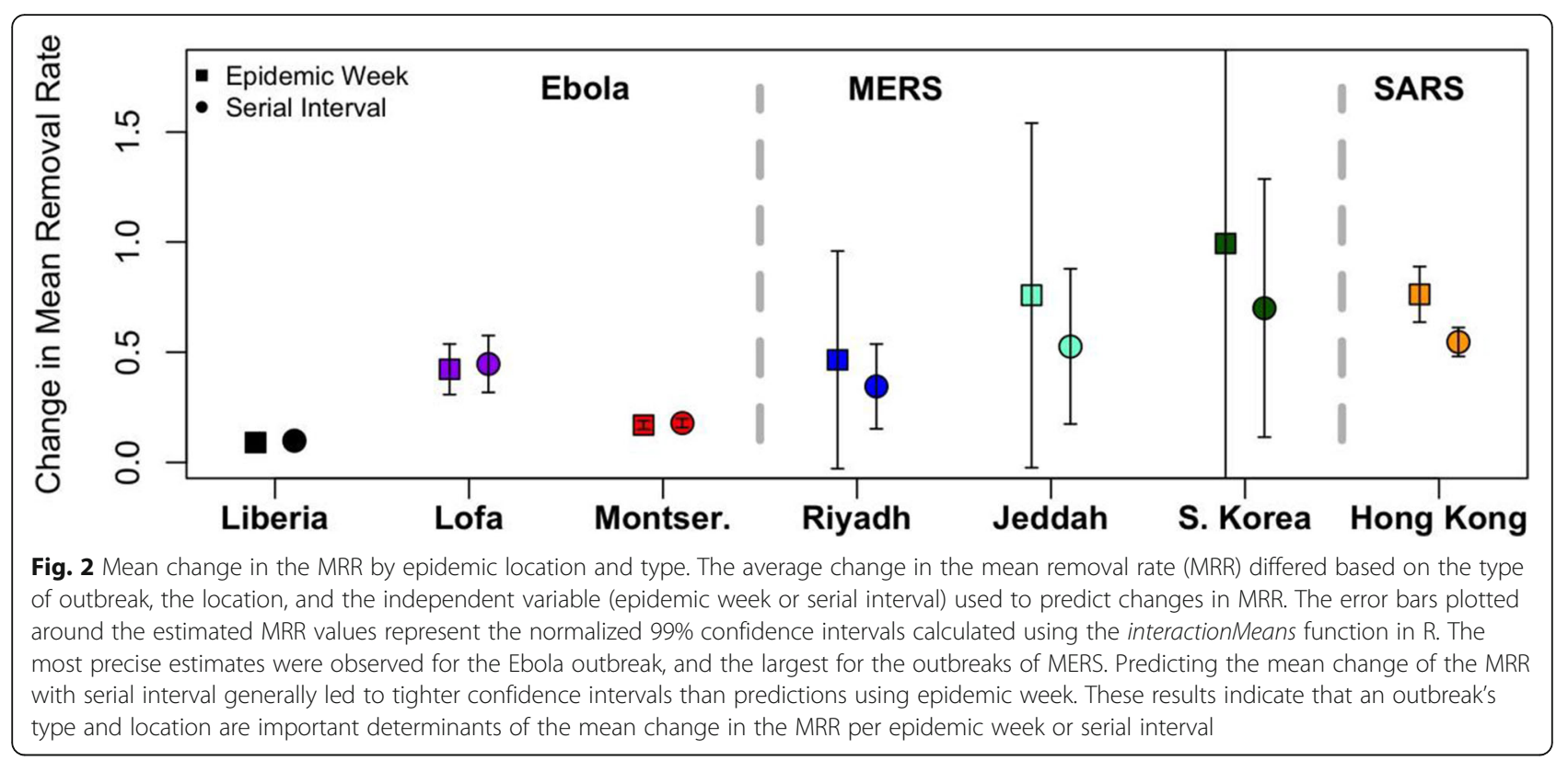

workers, access to health services, and density and frequency of social interaction. Comparing findings between different viral epidemics is also challenged by different periods and mechanisms of viral transmission (e.g. respiratory droplets versus aerosols, presence or absence of transmissibility before symptoms, etc.).

While our findings demonstrate large and statistically significant differences in MRR, it is notable that the calculated rates of change in the MRRs are within a factor of ten (when calculated using epidemic week) to seven (when calculated using serial interval) of each other (Fig. 2), with the mean change being the lowest in the EVD outbreak in Liberia and the highest in the MERS outbreak in South Korea. For modelers seeking to understand the epidemiology of emerging infectious diseases with limited or no data from previous outbreaks, this study provides a range of acceptable values for the MRR based on seven geographically distinct outbreaks of three emerging diseases. Similarly, while large disparities in DSOH are obvious (Fig. 1), these data highlight that all societies quickly adapt to outbreaks of emerging infections. Drake et al previously demonstrated the positive impact of behavior change in infectious outbreaks, noting that doubling the rate of "societal learning" in a model of the 2003 SARS outbreak in Singapore approximately halved the estimated number of infected patients [9]. While there is a theoretical upper limit to the speed with which newly-infected individuals can be removed from the susceptible population [9], public health strategies aimed at fostering behavioral adaptations and accelerating isolation should form a cornerstone of interventions tasked with limiting the spread of highly contagious and deadly emerging pathogens [31]. Such strategies extend beyond what we have presented here, such as prompt isolation of infected individuals within hospitals and other health care settings, and include other collective measures such as household quarantine practices, remote work, cancellation of mass gatherings, limitations on travel, use of facemasks or other virusspecific barriers against transmission, immunizations (when available), and more.

\section{Conclusions}

We have shown that rates of hospital or healthcare facility isolation of infected individuals from the susceptible population vary significantly by pathogen and location, but can in some cases be predicted by the timing and serial interval of the epidemic. This study detected variation in DSOH and MRR based on epidemic location and outbreak type, indicating that it may be possible to estimate a general range of the rate of change in these variables over time. Due to location-specific differences in DSOH and MRR, modelers who seek to develop forecasts early in an outbreak would benefit from estimating an expected range for removal of infected individuals using data from past outbreaks of the same pathogen in a similar setting. Furthermore, the quality of these estimates will be impacted by the metric chosen, as seen by the notable, but distinct, trends detected in DSOH and MRR. As seen in this study, even a relatively small amount of data from an emerging or reemerging outbreak of infectious disease can be paired with a well-chosen response variable such as $\mathrm{DSOH}$ or MRR to make effective forecasts about public health behavior. 


\section{Abbreviations}

EVD: Ebola Virus Disease; SARS: Severe Acute Respiratory Syndrome; MERS: Middle East Respiratory Syndrome; DSOH: Days from Symptom Onset to Hospitalization; MRR: Mean Removal Rate; SIR: Susceptible-InfectedRecovered; SIS: Susceptible-Infected-Susceptible

\section{Supplementary Information}

The online version contains supplementary material available at https://doi. org/10.1186/s12879-021-06299-x.

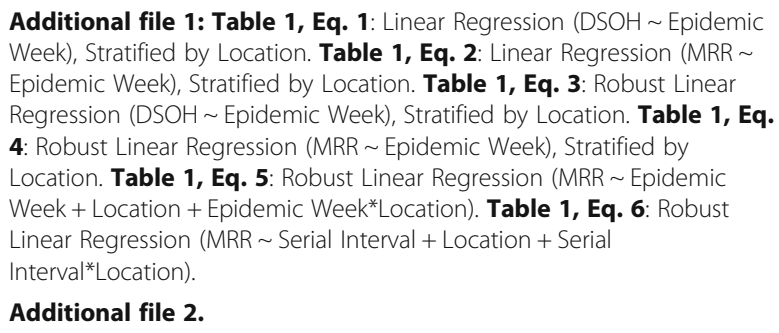

\section{Acknowledgements}

Not applicable.

\section{Authors' contributions}

EL helped develop the project and conduct initial data analysis with JD as part of the University of Georgia Population Biology of Infectious Diseases REU Site. AS updated EL's analyses with finalized Ebola data and wrote an initial paper draft from that work. EL and JD finalized the manuscript for submission. All authors read and approved the manuscript for submission.

\section{Funding}

This work was performed as part of the Population Biology of Infectious Diseases REU Site Program. Funding was provided by grants from the National Science Foundation (DBI-1156707) and the National Institute of General Medical Sciences of the National Institutes of Health under Award Number U01GM110744. Lodge was additionally supported by the University of North Carolina at Chapel Hill Medical Scientist Training Program (T32 GM008719-18), the National Institute of Environmental Health Sciences (T32 ES007018), and the National Institute of Child Health and Human Development (T32 HD007168). The content is solely the responsibility of the authors and does not necessarily reflect the official views of the National Science Foundation or the National Institutes of Health.

\section{Availability of data and materials}

We studied seven outbreaks: the 2013-2016 Liberian Ebola epidemic on a country-wide level, subsets of the same epidemic in Lofa and Montserrado Counties, the 2003 Hong Kong SARS epidemic, the 2014 Saudi Arabia MERS outbreaks in Riyadh and Jeddah, and the 2015 South Korea MERS outbreak. The Ebola data was originally obtained by the World Health Organization and provided by Christopher Dye (dyec@who.int). The Hong Kong SARS data was provided by Gabriel Leung (gmleung@hku.hk) of Hong Kong University. Please contact Christopher and Gabriel for data regarding Ebola and SARS, respectively, due to concerns regarding potentially identifiable health information. Finally, the MERS data for Saudi Arabia and South Korea were obtained from data compiled by Andrew Rambaut (a.rambaut@ed.ac.uk) of the University of Edinburgh, and is publicly available at https://github.com/ rambaut/MERS-Cases/blob/gh-pages/data/cases.csv. The code for this project was written by Annakate Schatz and Evans Lodge, and is available in the accompanying R Markdown file, Lodge_et_al_Behavior_Change.Rmd.

\section{Declarations}

\section{Ethics approval and consent to participate}

The Institutional Review Board (IRB) of the University of Georgia determined this study to be exempt (Protocol ID\#STUDY00002338) and waived requirements for participant consent. Data access permissions were granted by Christopher Dye (World Health Organization) for Ebola, Gabriel Leung (Hong Kong University) for SARS, and Andrew Rambaut (University of
Edinburgh) for MERS. All data was anonymized prior to access and use. See "Availability of Data and Materials" for more information.

\section{Consent for publication}

Not applicable.

\section{Competing interests}

The authors declare they have no actual or potential competing financial interests. Funders of this project played no role in data collection, study design, analysis, or manuscript preparation.

\section{Author details}

${ }^{1}$ Department of Epidemiology, Gillings School of Global Public Health, University of North Carolina at Chapel Hill, 135 Dauer Drive, Chapel Hill, NC 27599, USA. ${ }^{2}$ School of Medicine, University of North Carolina at Chapel Hill, Chapel Hill, North Carolina, USA. ${ }^{3}$ Odum School of Ecology and Center for Ecology of Infectious Diseases, University of Georgia, Athens, GA, USA.

Received: 28 April 2020 Accepted: 9 June 2021

Published online: 15 June 2021

\section{References}

1. Ebola Response Team WHO. Ebola virus disease in West Africa — the first 9 months of the epidemic and forward projections. N Engl J Med. 2014; 371(16):1481-95. https://doi.org/10.1056/NEJMoa1411100.

2. Garske T, Cori A, Ariyarajah A, Blake IM, Dorigatti I, Eckmanns T, et al. Heterogeneities in the case fatality ratio in the west African Ebola outbreak 2013-2016. Philos Trans R Soc B Biol Sci. 2017;372(1721):20160308. https:// doi.org/10.1098/rstb.2016.0308.

3. Ebola Response Team WHO. West African Ebola epidemic after one year slowing but not yet under control. N Engl J Med. 2015;372(6):584-7. https:// doi.org/10.1056/NEJMc1414992.

4. Dalziel BD, Kissler S, Gog JR, Viboud C, Bjørnstad ON, Metcalf CJE, et al. Urbanization and humidity shape the intensity of influenza epidemics in $U$. S. cities. Science. 2018;362(6410):75-9. https://doi.org/10.1126/science.aat603 0 .

5. Keeling MJ, Rohani P. Modeling infectious diseases in humans and animals. Princeton: Princeton University Press; 2011. 368 p. https://doi.org/10.2307/j. ctvcm4gk0.

6. Gray A, Greenhalgh D, Hu L, Mao X, Pan J. A stochastic differential equation SIS epidemic model. SIAM J Appl Math. 2011;71(3):876-902. https://doi.org/1 $0.1137 / 10081856$ X

7. Legrand J, Grais RF, Boelle PY, Valleron AJ, Flahault A. Understanding the dynamics of Ebola epidemics. Epidemiol Infect. 2007;135(04):610-21. https:// doi.org/10.1017/S0950268806007217.

8. $\quad$ Brug J, Aro AR, Richardus JH. Risk perceptions and behaviour: towards pandemic control of emerging infectious diseases. IntJ Behav Med. 2009; 16(1):3-6. https://doi.org/10.1007/s12529-008-9000-x.

9. Drake JM, Chew SK, Ma S. Societal learning in epidemics: intervention effectiveness during the 2003 SARS outbreak in Singapore. PLoS One. 2006; 1(1):e20. https://doi.org/10.1371/journal.pone.0000020.

10. Hayashi MAL. Integrating mathematical models of behavior and infectious disease: applications to outbreak dynamics and control: The University of Michigan; 2016

11. Freimuth $\mathrm{V}$, Linnan HW, Potter $\mathrm{P}$. Communicating the threat of emerging infections to the public. Emerg Infect Dis. 2000;6(4):337-47. https://doi.org/1 0.3201/eid0604.000403.

12. McLean KE, Abramowitz SA, Ball JD, Monger J, Tehoungue K, McKune SL, et al. Community-based reports of morbidity, mortality, and health-seeking behaviours in four Monrovia communities during the west African Ebola epidemic. Global Public Health. 2018;13(5):528-44. https://doi.org/10.1080/1 7441692.2016.1208262

13. Sebastian F, Iza C, Amanda T, Etienne G, Anton C, Eggo Rosalind M, et al. The impact of control strategies and behavioural changes on the elimination of Ebola from Lofa County, Liberia. Philos Trans R Soc B Biol Sci. 2017:372(1721):20160302.

14. Dimitri N. The economics of epidemic diseases. PLoS One. 2015;10(9): e0137964. https://doi.org/10.1371/journal.pone.0137964.

15. Regules JA, Beigel JH, Paolino KM, Voell J, Castellano AR, Hu Z, et al. A Recombinant Vesicular Stomatitis Virus Ebola Vaccine. N Engl J Med. 2017; 376(4):330-41. https://doi.org/10.1056/NEJMoa1414216. 
16. World Health Organization. Preliminary results on the efficacy of rVSVZEBOV-GP Ebola vaccine using the ring vaccination strategy in the control of an Ebola outbreak in the Democratic Republic of the Congo: an example of integration of research into epidemic response. 2019 [cited 2019 Aug 1]; Available from: https://www.who.int/csr/resources/publications/ebola/ebolaring-vaccination-results-12-april-2019.pdf?ua=1

17. Wells C, Yamin D, Ndeffo-Mbah ML, Wenzel N, Gaffney SG, Townsend JP, et al. Harnessing Case Isolation and Ring Vaccination to Control Ebola. PLoS Negl Trop Dis [Internet]. 2015 [cited 2019 Aug 1];9(5). Available from: https://www.ncbi.nlm.nih.gov/pmc/articles/PMC4449200/

18. Hong $\mathrm{S}$, Collins A. Societal responses to familiar versus unfamiliar risk: comparisons of influenza and SARS in Korea. Risk Anal. 2006;26(5):1247-57. https://doi.org/10.1111/j.1539-6924.2006.00812.x.

19. Kerstiëns B, Matthys F. Interventions to Control Virus Transmission during an Outbreak of Ebola Hemorrhagic Fever: Experience from Kikwit, Democratic Republic of the Congo, 1995. J Infect Dis. 1999;179(Supplement 1):S263-7.

20. Ngwa GA, Teboh-Ewungkem MI. A Mathematical Model with Quarantine States for the Dynamics of Ebola Virus Disease in Human Populations. Comput Math Methods Med [Internet]. 2016; [cited 2019 Jul 11];2016. Available from: https://www.ncbinlm.nih.gov/pmc/articles/PMC4992550/.

21. Hsieh Y-H, King C-C, Chen CWS, Ho M-S, Hsu S-B, Wu Y-C. Impact of quarantine on the 2003 SARS outbreak: a retrospective modeling study. J Theor Biol. 2007;244(4):729-36. https://doi.org/10.1016/j.jtbi.2006.09.015.

22. Bauch CT, Galvani AP. Social factors in epidemiology. Science. 2013; 342(6154):47-9. https://doi.org/10.1126/science.1244492.

23. Chowell G, Nishiura H. Transmission dynamics and control of Ebola virus disease (EVD): a review. BMC Med. 2014;12(1):196. https://doi.org/10.1186/ s12916-014-0196-0.

24. Wallinga J, Teunis P. Different epidemic curves for severe acute respiratory syndrome reveal similar impacts of control measures. Am J Epidemiol. 2004; 160(6):509-16. https://doi.org/10.1093/aje/kwh255.

25. Riley S, Fraser C, Donnelly CA, Ghani AC, Abu-Raddad LJ, Hedley AJ, et al. Transmission dynamics of the etiological agent of SARS in Hong Kong: impact of public health interventions. Science. 2003;300(5627):1961-6. https://doi.org/10.1126/science.1086478.

26. Cauchemez S, Boëlle P-Y, Donnelly CA, Ferguson NM, Thomas G, Leung GM, et al. Real-time estimates in early detection of SARS. Emerg Infect Dis. 2006; 12(1):110-3. https://doi.org/10.3201/eid1201.050593.

27. Assiri A, McGeer A, Perl TM, Price CS, Al Rabeeah AA, Cummings DAT, et al. Hospital outbreak of Middle East respiratory syndrome coronavirus. N Engl J Med. 2013;369(5):407-16. https://doi.org/10.1056/NEJMoa1306742.

28. Cowling BJ, Park M, Fang VJ, Wu P, Leung GM, Wu JT. Preliminary epidemiologic assessment of MERS-CoV outbreak in South Korea, May-June 2015. Euro Surveill [Internet]. 2015;20(25) [cited 2019 Jul 30], Available from: https:/www.ncbi.nlm.nih.gov/pmc/articles/PMC4535930/

29. Bland JM, Altman DG. Multiple significance tests: the Bonferroni method. BMJ. 1995;310(6973):170. https://doi.org/10.1136/bmj.310.6973.170.

30. R Development Core Team. R: a language and environment for statistical computing [internet]. Vienna: the R Foundation for statistical Computing; 2011. Available from: http://www.R-project.org/

31. Barbisch D, Koenig KL, Shih F-Y. Is there a case for quarantine? Perspectives from SARS to Ebola. Disaster Med Public Health Preparedness. 2015;9(5): 547-53. https://doi.org/10.1017/dmp.2015.38.

\section{Publisher's Note}

Springer Nature remains neutral with regard to jurisdictional claims in published maps and institutional affiliations.

Ready to submit your research? Choose BMC and benefit from:
- fast, convenient online submission
- thorough peer review by experienced researchers in your field
- rapid publication on acceptance
- support for research data, including large and complex data types
- gold Open Access which fosters wider collaboration and increased citations
- maximum visibility for your research: over 100M website views per year
At BMC, research is always in progress.
Learn more biomedcentral.com/submissions

